\title{
Primary Corneal Squamous Cell Carcinoma in a Dog: Clinical and Histopathological Evaluation
}

\author{
Giovanni Barsotti, ${ }^{1}$ Lorenzo Ressel, ${ }^{2}$ Riccardo Finotello, ${ }^{3}$ \\ Veronica Marchetti, ${ }^{1}$ and Francesca Millanta ${ }^{2}$ \\ ${ }^{1}$ Department of Veterinary Clinics, Faculty of Veterinary Medicine, University of Pisa, Via Livornese Lato Monte, \\ San Piero a Grado, 56122 Pisa, Italy \\ ${ }^{2}$ Department of Animal Pathology, Faculty of Veterinary Medicine, University of Pisa, Delle Piagge 2 Avenue, 56100 Pisa, Italy \\ ${ }^{3}$ Small Animal Teaching Hospital, University of Liverpool, Leahurst, Liverpool, Leahurst Campus Chester High Road, \\ Neston, Wirral CH64 7TE, UK
}

Correspondence should be addressed to Giovanni Barsotti, gbarsott@vet.unipi.it

Received 21 November 2011; Accepted 16 January 2012

Academic Editor: M. Bugno-Poniewierska

Copyright (c) 2012 Giovanni Barsotti et al. This is an open access article distributed under the Creative Commons Attribution License, which permits unrestricted use, distribution, and reproduction in any medium, provided the original work is properly cited.

\begin{abstract}
An 8-year-old male pug with a 12-month history of a progressive nonpainful mass on the left cornea was evaluated. Ocular examination showed a severe bilateral keratoconjunctivitis sicca, pigmentary keratitis, and an exophytic irregular pink mass occupying approximately $75 \%$ of the total corneal surface of the left eye. A squamous cell carcinoma (SCC) was suspected on cytology, and clinical investigations showed no evidence of metastases. A transpalpebral enucleation was therefore performed, and the diagnosis of SCC was confirmed on histopathology. Immunohistochemical investigations showed that the neoplastic cells were pan-cytokeratin positive and vimentin negative. Additionally, nuclei immunoreactive to Ki-67 antigen were detected. Tumor cells were also negative to $\mathrm{p} 53$. Immunoreactivity to COX-2 was found in less than $10 \%$ of the neoplastic cells. No adjuvant therapies were instituted, and no evidence of local recurrence or distance metastasis was identified during the 24-month follow-up period.
\end{abstract}

\section{Introduction}

Neoplasms of the cornea occur uncommonly in dogs although various primary and secondary tumors have been described in veterinary literature $[1,2]$. Corneal squamous cell carcinoma (SCC) is considered rare in the dog and often represents a secondary extension of a primary limbal or conjunctival neoplasia $[1,3,4]$. A number of cases of canine primary SCC of the cornea have been described especially in recent years [5-10], and some authors report an increased occurrence of the tumor in this period [11]. Only three canine SCCs have been characterized by the use of immunohistochemistry, and $\mathrm{p} 53$ protein was generally investigated with varying results $[7,9]$. Cyclooxygenase (COX) overexpression has been identified in various neoplastic tissues in humans [12-18] and domestic animals [19-26]. COX-2 has been found to be strongly expressed in all the cases of canine SCC of the cutis [27].
Since the etiopathogenesis of ocular SCC is still unclear, some authors have evaluated the expression of COX, especially COX-2, in corneal neoplastic tissues of horses and have suggested a possible role of the enzyme in oncogenesis and/or progression of this type of corneal tumor [23, 24].

The aim of the present study was to describe the clinical and histopathological appearance of a primary corneal SCC in an 8 year-old male pug dog, to report its histopathological findings, and to characterise the tumour using antivimentin and antipan cytokeratin antibodies and evaluate the expression of cyclooxygenase-2, p53 protein, and Ki-67 antigen in the neoplastic cornea by the use of immunohistochemical (IHC) techniques.

\section{Case Presentation}

An 8 year-old, $8 \mathrm{~kg}$, intact male Pug was examined for 12month history of a progressive nonpainful lesion of the 


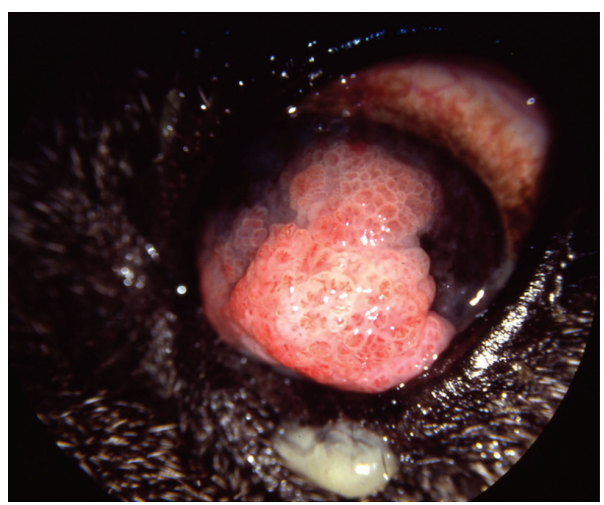

(a)

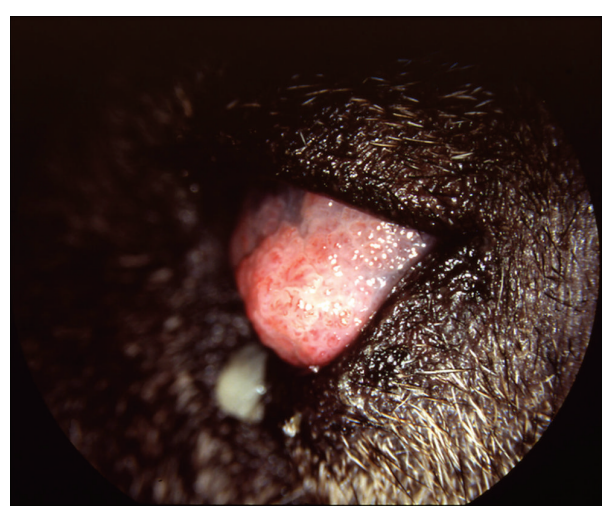

(b)

Figure 1: (a) Photograph of the left eye of an 8-year-old male Pug demonstrating an exophytic, irregular reddish-pink mass occupying approximately $75 \%$ of the total corneal surface. The cornea surrounding the mass is pigmented and vascularized. (b) Lateral view of the eye. Note the highly deformed profile of the cornea.

left cornea. The past medical record of the dog excluded any major illnesses, vaccinations were routinely performed, and the dog was on heartworm prophylaxis. Serology for Leishmania infantum was performed every year with negative results. Physical examination revealed no other abnormal findings.

Gross ocular examination showed a bilateral nasal fold trichiasis, a bilateral ocular discharge, an exophytic reddishpink mass arising from the cornea of the left eye, and a complete corneal, pigmentation of the right eye. Schirmer I tear test readings obtained using commercially available test strips (Dina strip Schirmer-Plus; GECIS sarl, France) were 3 and $4 \mathrm{~mm} / \mathrm{min}$ OS and OD, respectively. Palpebral, corneal and dazzle reflexes were present in both eyes (OU), whereas menace response was negative in both eyes. Because of the complete opacity of the cornea of both eyes, it was no possible to evaluate direct and indirect pupillary light reflexes. The intraocular pressure was assessed by means of applanation tonometry (Tonopen-XL; Mentor, MA, USA) following a topical administration of $0.4 \%$ oxybuprocaine chlorhydrate (Benoxinato chlorhydrate Intes; Alfa Intes Industria Terapeutica Splendore S.r.l., Italy) and was found to be $18 \mathrm{mmHg}$ in the right eye. The highly deformed profile of the left cornea did not allow us to obtain reliable intraocular pressure values from the left eye. Slit-lamp biomicroscopy (Kowa SL-14, Kowa company, Japan) showed conjunctival hyperemia and mild chemosis OU, a complete pigmentation of the right cornea with neovascularization and an exophytic, irregular reddish-pink mass occupying approximately 75\% of the total corneal surface of the left eye (Figure 1). The growth was also firm, moderately friable, and bled easily on manipulation. The peripheral cornea, not involved by the lesion, was pigmented and vascularized, and the limbus and sclera were unaffected. The anterior chamber, the iris, the vitreous, and the fundus were not examined OU because of the complete opacity of both corneas.

An initial diagnosis of a suspected corneal tumor of the left eye, bilateral keratoconjunctivitis sicca, and pigmentary keratitis was formulated.

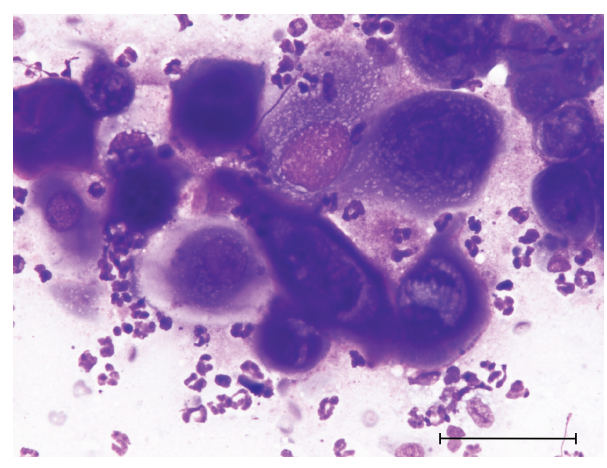

FIgUre 2: Cytological features. Pleomorphic squamous epithelium with marked anisokaryosis, hyperbasophilic cytoplasm, and perinuclear vacuolation associated with neutrophilic inflammation (magnification bar = 50 microns, May-Grunwald Giemsa stain).

Differential diagnosis of the mass included primary corneal tumor and nonneoplastic corneal lesions such as granulation tissue and chronic proliferative keratitis.

The diagnostic workup included CBC, blood smear evaluation, serum biochemical profile, coagulation profile, urinalysis, and fine needle aspiration biopsy (FNAB) of the mass. CBC, serum biochemical profile, coagulation profile, and urinalysis results were unremarkable.

Cytologic specimens were stained with May-Grunwald Giemsa and showed a moderate blood contamination and rounded, spindled, and angulated cells. Cells with hyperbasophilic keratinized cytoplasm displayed anisokaryosis and perinuclear vacuolization. Nondegenerate neutrophils were also present (Figure 2). The cytological pattern was compatible with an epithelial tumour and strongly suspicious of a squamous cell carcinoma.

The dog was then staged to evaluate the presence of distance metastases. An ocular ultrasound of the left eye, performed to rule out any orbital and intraocular involvement, was negative. Chest X-ray in three standard projections and abdominal ultrasonography were performed, revealing no evidence of disease. 


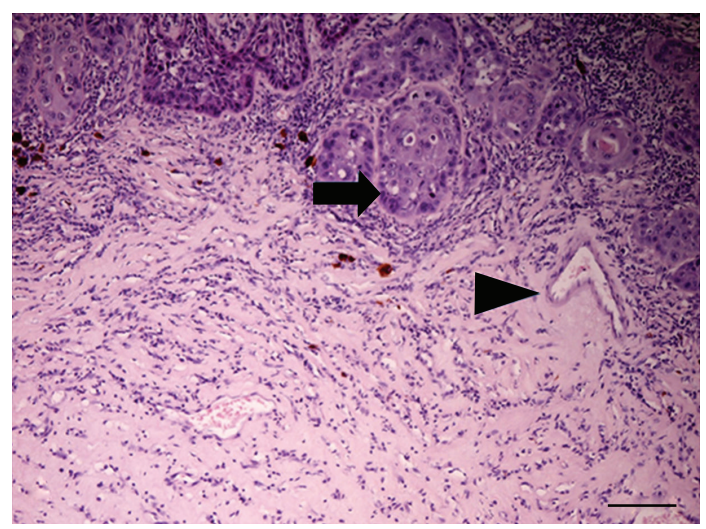

(a)

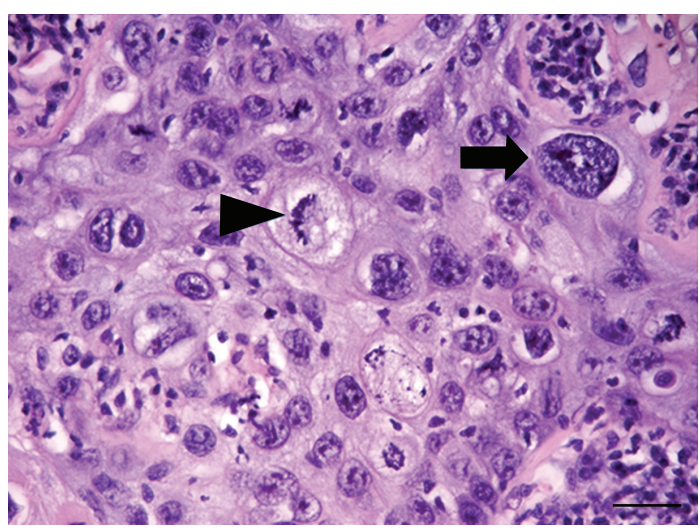

(b)

Figure 3: Histopathological features. (a) Proliferation and invasion of the stroma by epithelial neoplastic cells (arrow). A blood vessel is present in the stroma (arrowhead) (magnification bar $=100$ microns, haematoxylin and eosin stain). (b) Neoplastic epithelial cells are characterized by atypical anisokaryotic nuclei (arrow) and frequent mitotic figures (arrowhead) (magnification bar $=25$ microns, haematoxylin and eosin stain).

Considering the clinical aspect of the lesion, the results of the FNAB and the concurrent severe keratoconjunctivitis sicca, a transpalpebral enucleation of the left eye was performed. After surgery, the dog recovered well from anaesthesia, and no adjuvant therapies were instituted. Followup every six months did not reveal any sign of orbital recurrence or metastatic disease. The dog appeared to be sound at the last clinical staging performed 24 months after surgery.

The enucleated eye was fixed in a 10\% formalin solution before being routinely processed and paraffin-wax embedded. Sections were stained with haematoxylin and eosin (HE). Microscopically, the corneal epithelium was focally affected by an unencapsulated proliferation of squamous epithelial corneal cells infiltrating the underlying connective tissue (Figure 3(a)). Neoplastic cells were arranged in nests (Figure 3(a), arrow) and in multifocally formed foci of intraepithelial keratinisation. Neoplastic cells were round to polygonal, pleomorphic with abundant cytoplasm, with irregularly shaped immature nuclei characterized by a high grade of anisokaryosis and multiple nucleoli (Figure 3(b), arrow). Mitotic figures were frequent (45 per $10 \mathrm{HPFs}$ ), and often bizarre (Figure 3(b), arrowhead). The neoplasia showed an inflammatory cell infiltrate composed of lymphocytes and neutrophils, and the infiltrated stroma was characterized by the presence of newly formed blood vessels (Figure 3(a), arrowhead).

To perform IHC analysis, the sections were deparaffinized in Bio-Clear (Bio-Optica, Milan, Italy) and hydrated with grade ethanol concentration until distilled water. Antigen retrieval was performed by calibrated water bath capable of maintaining the epitope retrieval solution in a $10 \mathrm{mM}$ sodium citrate buffer ( $\mathrm{pH} 6.0$ ) at $97^{\circ} \mathrm{C}$ for $30 \mathrm{~min}$. The sections were allowed to cool down at room temperature (RT) for $20 \mathrm{~min}$. To block endogenous peroxidase activity, the slides were treated with $3.0 \%$ hydrogen peroxide in distilled water for $10 \mathrm{~min}$ and washed with phosphatebuffered saline (PBS; Dako, Denmark) two or three times.

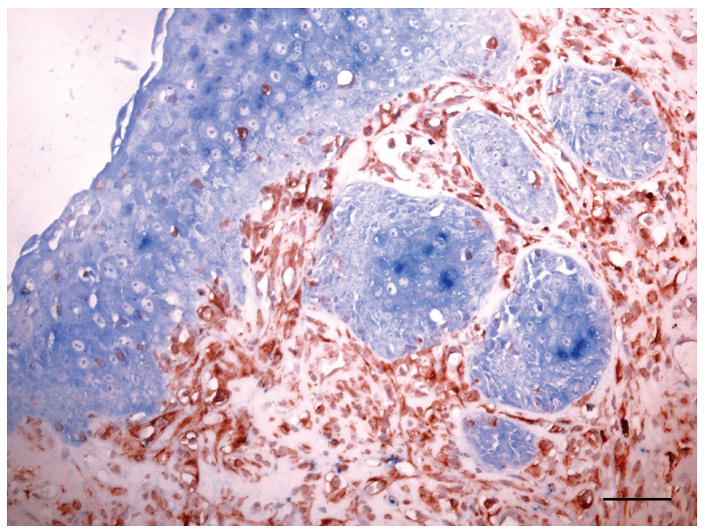

FIGURE 4: Immunohistochemical staining. Neoplastic epithelial cells express Pan-Cytokeratin (blue stain) and invade the stroma (brown stain) (magnification bar $=25$ microns, double immunohistochemistry).

After blocking nonspecific antigen with normal horse serum (UltraVision; LabVision, CA, USA), the following was used as primary commercial antibodies: anti-COX-2 (goat polyclonal; Santacruz biotech, CA, USA) at $1: 100$ dilution, antiKi-67 (clone MIB-1; Neomarkers, CA, USA) at $1: 100$ dilution, anti-Pan-Cytokeratin (rabbit polyclonal; CKp Novocastra Laboratories, Newcastle upon Tyne, UK), antivimentin (clone V9; Dako, Denmark) at 1:100 dilution, and antip53 (mouse monoclonal clone DO7; Dako, Denmark) at dilution $1: 25$. The incubation of the primary antibodies was performed at room temperature for 60 minutes. The IHC analyses were performed using the streptavidin-biotin alkaline phosphatase complex (UltraVision; LabVision, CA, USA), and the peroxidase reaction was developed for $10 \mathrm{~min}$ with 3,3'-diaminobenzidine (DAB; Vector Laboratories Inc., CA, USA) and stopped with deionised water. The sections were counterstained with Mayer's hematoxylin. Negative controls were performed by substituting the primary antibody with a nonimmune serum at the same concentration. 


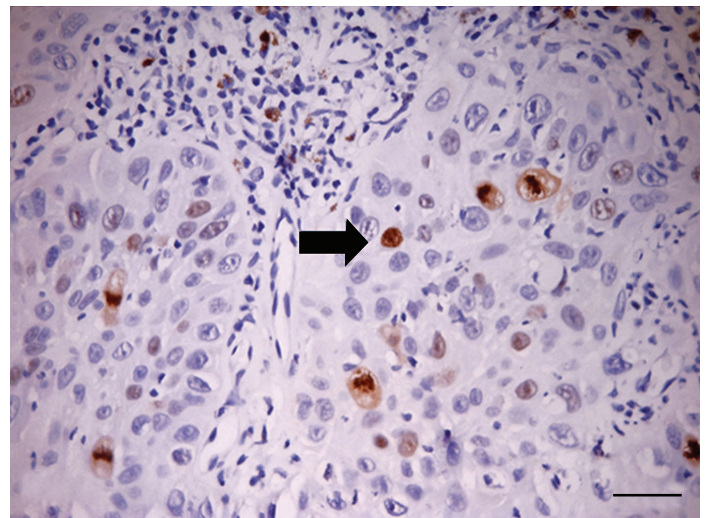

(a)

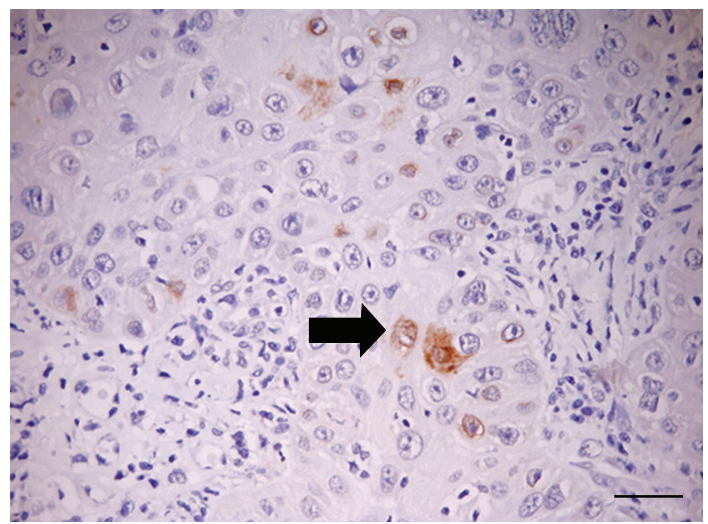

(b)

FIGURE 5: Immunohistochemical staining. (a) Ki67-positive cells (arrow) in the context of epithelial neoplastic cells (magnification bar $=$ 25 microns, immunohistochemistry). (b) A small percentage of neoplastic cells express COX-2 (arrow) (magnification bar $=25$ microns, immunohistochemistry).

COX-2 immunoexpression was quantified according to previously established guidelines [22]. The labeling index of Ki-67 was quantified as previously described [28]. Double immunohistochemistry was performed to investigate pancytokeratin and vimentin simultaneous expression using Multivision system (Thermo Scientific, MA, USA), following manufacturer's instructions.

Double immunostaining for pan-cytokeratin and vimentin showed a pan-cytokeratin positive/Vimentin negative pattern of tumor cells (Figure 4) and allowed their differentiation from stromal cells that expressed Vimentin alone. Cell proliferation, assessed by Ki-67 labelling index, was 8 positive nuclei/1000 cells at 400x (Figure 5(a)). Less than 10\% of neoplastic cells were positive for COX-2 immunostaining (Figure 5(b)), and thus the tumor was considered as nonCOX-2 overexpressing. No immunoreactivity to p53 was observed.

\section{Discussion}

Primary corneal squamous cell carcinoma (SCC) is considered rare in dogs $[1,2]$, indeed, until 2008, only four cases of this type of tumor had been described $[5,6]$. Nevertheless, in the last two years, some case reports $(n=6)[7-10]$ and a case series $(n=26)$ [11] of canine primary corneal SCC have been published, and occurrence of this type of tumor seems to be increasing [11]. Most of the dogs presenting with corneal SCC had had a history of chronic keratitis [11], such as keratoconjunctivitis sicca [6], pigmentary keratitis $[6,9]$ or keratitis secondary to eyelid abnormalities [7], or trauma [5]. Only a few dogs did not show any concurrent ocular surface disease at the time of the diagnosis $[8,11]$. Considering the clinical evidence of chronic keratitis in most cases, a continuous ocular surface inflammation seems to play a crucial role in the development of primary corneal SCC in dogs. Likewise, the object of this study presented a severe bilateral keratoconjunctivitis sicca and pigmentary keratitis with a 2-year history of mucous ocular discharge and corneal opacity with no record of topical treatment.
With respect to breed predisposition, brachycephalic dogs, especially pugs, with a history of chronic keratitis seem to be predisposed to developing this type of tumor. Out of 36 dogs presenting a primary corneal SCC described in veterinary literature [5-11], 13 were pugs, as is the dog of the present case report. Pugs exhibit a certain degree of exophthalmos, macroblepharon, and lagophthalmos, and this combination of anatomic features causes inadequate blinking and leads to exposure keratopathy syndrome. In addition, many pugs show a nasal fold trichiasis and a mild medial entropion of the lower eyelid. The severe corneal irritation secondary to its exposure, to eyelid defects, and to the presence of facial folds might explain the high prevalence of primary SCC cases in this breed.

Some authors have reported a possible relationship between the occurrence of corneal SCC and the use of topical immunosuppressants [11], but it is important to note that all the dogs who received this type of drug also showed a chronic keratitis. Thus, it is difficult to establish which is the most likely predisposing factor (chronic corneal inflammation versus immunosuppressant drugs) in the development of neoplastic disease.

Primary corneal SCC might be a related consequence of ultraviolet radiation exposure as reported by MontianiFerreira et al. [7]. In fact the IHC results of their study detected a strong p53 expression that is considered suggestive of p53 gene mutation which is likely to be a consequence of ultraviolet radiation exposure [29]. P53 is a nuclear phosphoprotein that is normally present in various tissues. The wild type of this protein is characterized by a short half-life (20-30 $\mathrm{min}$ ) [30] and is usually undetectable with IHC [31]. If a somatic mutation occurs, the p53 half-life increases ( 2 hours) and the protein becomes detectable with IHC methods [30]. P53 mutation is a common feature of many neoplasms in both human and veterinary medicine, and p53 changes have also been observed in ocular SCC of domestic animals [32-35], and human beings [36].

Our results showed the absence of p53 immunoreactivity, in accordance with Takiyama et al. [9] who described two cases of canine primary corneal SCC in which nuclear p53 
immunoreactivity was not detected. The adopted antibody, the same as used by Takiyama et al., was the monoclonal (MAb) mouse antihuman p53 protein clone DO-7. Instead, Montiani-Ferreira et al. [7] observed a strong p53 expression in the neoplastic tissue using a rabbit polyclonal (PAb) antihuman p53 protein. Differences between the results of Montiani-Ferreira et al. [7], and Takiyama et al. [9] and ours could be related to the use of different antibodies and staining protocols. Another possible cause explaining the negativity for p53 staining is a deletion of the p53 gene resulting in the absence of $\mathrm{p} 53$ protein in neoplastic cells. On the other hand, the negativity for p53 could also be related to no mutation of this protein, and thus, in our case, p53 gene mutation due to UV overexposure may not be a predisposing factor in the occurrence of the primary corneal SCC. It is important to note that Italy has UV index ranges 1-3 in January and 7-9 in July, slightly higher than Japan's [9] but lower than Brazil's, where the monthly average UV index goes from 3.5 in February to 13.4 in July [7]. In Italy, as in Japan, there is a lower UV exposure risk and probably a lower UV influence in corneal SCC development. This latter hypothesis may also explain the low Ki-67 labelling index observed. The lack of p53 mutations may, in fact, not have altered the cell-cycle regulation. It is well known that $\mathrm{Ki}$ 67 is a nonhistone nuclear protein which is expressed in all the phases of the cell cycle except G0 and G1, and that it is, therefore, widely used to assess the cell proliferation [37].

COX-2 is the inducible form of COX and catalyzes the production of prostaglandins from arachidonic acid. Of these prostaglandins, $\mathrm{PGE}_{2}$ is the one primarily responsible for several tumorigenic effects, such as the increase of cell proliferation, inhibition of apoptosis, and neoangiogenesis. Our case showed a weak and focal immunoreactivity to COX-2 in less than $10 \%$ of neoplastic cells and was considered as a non-COX-2-overexpressing tumor [22, 38-40]. Previous studies have described COX-2 expression in SCC of the cornea in horses $[23,24]$. Since the methodologies, as well as the scoring methods, adopted are different, it is still unclear whether COX-2 plays a role in the development and progression of this type of corneal tumor. A recent study performed by Smith et al. [25] has shown that COX-2 was expressed in a low percentage of tumor cells and at low intensity in equine ocular SCC.

Our results do not lend themselves as a rationale to consider COX-2 pharmacological inhibition as an adjuvant tool in the treatment of corneal SCC in dogs, as previously suggested by Smith et al. [25] in ocular SCC in horses.

In the case of dogs with normal function of the eye affected by corneal SCC, a superficial keratectomy alone $[4,6,9]$ or associated with adjuvant therapies (cryosurgery [5] or topical mitomycin C [8]) has been described with good results.

In our dog we performed a transpalpebral enucleation without adjuvant therapies, and we did not consider any treatment to preserve the affected eye because of the wide extension of the neoplasia above the cornea, but especially because of the concurrent presence of a severe keratoconjunctivitis sicca, which could have delayed corneal healing with postoperative complications.
In conclusion, surgical treatment must be considered the best therapeutic approach for ocular SCC in dogs. Further studies would be useful to better understand the role of COX2 expression and its therapeutic implication in corneal SCC of the dog, especially in dogs where the conservative surgical treatment, superficial keratectomy, is chosen.

\section{References}

[1] C. A. Fischer, D. M. Lindley, W. C. Carlton, and H. van Hecke, "Tumors of the cornea and sclera," in Ocular Tumors in Animals and Humans, R. L. Peiffer Jr and K. B. Simons, Eds., pp. 149-202, Blackwell Publishing, Ames, Iowa, USA, 1st edition, 2002.

[2] B. C. Gilger, E. Bentley, and F. J. Ollivier, "Disease and surgery of the cornea and sclera," in Veterinary Ophthalmology, K. N. Gelatt, Ed., pp. 690-752, Blackwell Publishing, Ames, Iowa, USA, 4th edition, 2007.

[3] D. A. Ward, K. S. Latimer, and R. M. Askren, "Squamous cell carcinoma of the corneoscleral limbus in a dog," Journal of the American Veterinary Medical Association, vol. 200, no. 10, pp. 1503-1506, 1992.

[4] C. Busse, J. Sansom, R. R. Dubielzig, and A. Hayes, "Corneal squamous cell carcinoma in a Border Collie," Veterinary Ophthalmology, vol. 11, no. 1, pp. 55-58, 2008.

[5] K. S. Latimer, R. L. Kaswan, and J. P. Sundberg, "Corneal squamous cell carcinoma in a dog," Journal of the American Veterinary Medical Association, vol. 190, no. 11, pp. 14301432, 1987.

[6] M. E. Bernays, D. Flemming, and R. L. Peiffer, "Primary corneal papilloma and squamous cell carcinoma associated with pigmentary keratitis in four dogs," Journal of the American Veterinary Medical Association, vol. 214, no. 2, pp. 215-217, 1999.

[7] F. Montiani-Ferreira, M. Kiupel, P. Muzolon, and J. Truppel, "Corneal squamous cell carcinoma in a dog: a case report," Veterinary Ophthalmology, vol. 11, no. 4, pp. 269-272, 2008.

[8] K. Karasawa, H. Matsuda, and A. Tanaka, "Superficial keratectomy and topical mitomycin $\mathrm{C}$ as therapy for a corneal squamous cell carcinoma in a dog: case report," Journal of Small Animal Practice, vol. 49, no. 4, pp. 208-210, 2008.

[9] N. Takiyama, E. Terasaki, and M. Uechi, "Corneal squamous cell carcinoma in two dogs," Veterinary Ophthalmology, vol. 13, no. 4, pp. 266-269, 2010.

[10] F. S. Prando, F. G. Jannuzzi, M. Rosa, C. G. Lieberknecht, C. F. Lieberknecht, and J. S. Pereira, "Squamous cell carcinoma in the pug cornea-report of two cases (abstract)," Veterinary Ophthalmology, vol. 13, no. 5, p. 408, 2010.

[11] R. R. Dubielzig, C. S. Schobert, and J. Dreyfus, "Superficial corneal squamous cell carcinoma occurring in dogs with chronic keratitis," Veterinary Ophthalmology, vol. 14, no. 3, pp. 161-168, 2011.

[12] O. Gallo, E. Masini, B. Bianchi, L. Bruschini, M. Paglierani, and A. Franchi, "Prognostic significance of cyclooxygenase2 pathway and angiogenesis in head and neck squamous cell carcinoma," Human Pathology, vol. 33, no. 7, pp. 708-714, 2002.

[13] B. Singh, J. A. Berry, A. Shoher, V. Ramakrishnan, and A. Lucci, "COX-2 overexpression increases motility and invasion of breast cancer cells," International Journal of Oncology, vol. 26, no. 5, pp. 1393-1399, 2005.

[14] T. C. Tang, R. T. Poon, C. P. Lau, D. Xie, and S. T. Fan, "Tumor cyclooxygenase-2 levels correlate with tumor invasiveness in 
human hepatocellular carcinoma," World Journal of Gastroenterology, vol. 11, no. 13, pp. 1896-1902, 2005.

[15] R. Miao, N. Liu, Y. Wang et al., "Coexpression of cyclooxygenase-2 and vascular endothelial growth factor in gastrointestinal stromal tumor: possible relations to pathological parameters and clinical behavior," Hepato-Gastroenterology, vol. 55, no. 88, pp. 2012-2015, 2008.

[16] J. A. G. Filho, C. F. W. Nonaka, M. C. D. C. Miguel, R. D. A. Freitas, and H. C. Galvão, "Immunoexpression of cyclooxygenase-2 and p53 in oral squamous cell carcinoma," American Journal of Otolaryngology, vol. 30, no. 2, pp. 89-94, 2009.

[17] R. Kawata, S. Hyo, M. Araki, and H. Takenaka, "Expression of cyclooxygenase-2 and microsomal prostagalandin E synthase1 in head and neck squamous cell carcinoma," Auris Nasus Larynx, vol. 37, no. 4, pp. 482-487, 2010.

[18] H. U. Kasper, E. Konze, H. P. Dienes, D. L. Stippel, P. Schirmacher, and M. Kern, "COX-2 expression and effects of COX-2 inhibition in colorectal carcinomas and their liver metastases," Anticancer Research, vol. 30, no. 6, pp. 2017-2023, 2010.

[19] K. N. M. Khan, D. W. Knapp, D. B. Denicola, and R. K. Harris, "Expression of cyclooxygenase-2 in transitional cell carcinoma of the urinary bladder in dogs," American Journal of Veterinary Research, vol. 61, no. 5, pp. 478-481, 2000.

[20] K. N. M. Khan, K. M. Stanfield, D. Trajkovic, and D. W. Knapp, "Expression of cyclooxygenase-2 in canine renal cell carcinoma," Veterinary Pathology, vol. 38, no. 1, pp. 116-119, 2001.

[21] M. Kleiter, D. E. Malarkey, D. E. Ruslander, and D. E. Thrall, "Expression of cyclooxygenase-2 in canine epithelial nasal tumors," Veterinary Radiology and Ultrasound, vol. 45, no. 3, pp. 255-260, 2004.

[22] F. Millanta, S. Citi, D. D. Santa, M. Porciani, and A. Poli, "COX-2 expression in canine and feline invasive mammary carcinomas: correlation with clinicopathological features and prognostic fmolecular markers," Breast Cancer Research and Treatment, vol. 98, no. 1, pp. 115-120, 2006.

[23] C. L. McInnis, E. A. Giuliano, P. J. Johnson, and J. R. Turk, "Immunohistochemical evaluation of cyclooxygenase expression in corneal squamous cell carcinoma in horses," American Journal of Veterinary Research, vol. 68, no. 2, pp. 165-170, 2007.

[24] K. M. Rassnick and B. L. Njaa, "Cyclooxygenase-2 immunoreactivity in equine ocular squamous-cell carcinoma," Journal of Veterinary Diagnostic Investigation, vol. 19, no. 4, pp. 436-439, 2007.

[25] K. M. Smith, T. J. Scase, J. L. Miller, D. Donaldson, and J. Sansom, "Expression of cyclooxygenase-2 by equine ocular and adnexal squamous cell carcinomas," Veterinary Ophthalmology, vol. 11, no. 1, pp. 8-14, 2008.

[26] F. L. Queiroga, I. Pires, L. Lobo, and C. S. Lopes, "The role of Cox-2 expression in the prognosis of dogs with malignant mammary tumours," Research in Veterinary Science, vol. 88, no. 3, pp. 441-445, 2010.

[27] E. M. P. de Almeida, C. Piché, J. Sirois, and M. Doré, "Expression of cyclo-oxygenase-2 in naturally occurring squamous cell carcinomas in dogs," Journal of Histochemistry and Cytochemistry, vol. 49, no. 7, pp. 867-875, 2001.

[28] F. Millanta, G. Lazzeri, M. Mazzei, I. Vannozzi, and A. Poli, "MIB-1 labeling index in feline dysplastic and neoplastic mammary lesions and its relationship with postsurgical prognosis," Veterinary Pathology, vol. 39, no. 1, pp. 120-126, 2002.
[29] V. O. Melnikova, A. Pacifico, S. Chimenti, K. Peris, and H. N. Ananthaswamy, "Fate of UVB-induced p53 mutations in SKH-hrl mouse skin after discontinuation of irradiation: relationship to skin cancer development," Oncogene, vol. 24, no. 47, pp. 7055-7063, 2005.

[30] B. Vojtěsěk, J. Bàrtek, C. A. Midgley, and D. P. Lane, "An immunochemical analysis of the human nuclear phosphoprotein p53. New monoclonal antibodies and epitope mapping using recombinant p53," Journal of Immunological Methods, vol. 151, no. 1-2, pp. 237-244, 1992.

[31] K. Cooper and Z. Haffajee, "bcl-2 and p53 protein expression in follicular lymphoma," Journal of Pathology, vol. 182, no. 3, pp. 307-310, 1997.

[32] S. J. Dugan, C. R. Curtis, S. M. Roberts, and G. A. Severin, "Epidemiologic study of ocular/adnexal squamous cell carcinoma in horses," Journal of the American Veterinary Medical Association, vol. 198, no. 2, pp. 251-256, 1991.

[33] S. J. Dugan, S. M. Roberts, C. R. Curtis, and G. A. Severin, "Prognostic factors and survival of horses with ocular/adnexal squamous cell carcinoma: 147 cases (1978-1988)," Journal of the American Veterinary Medical Association, vol. 198, no. 2, pp. 298-303, 1991.

[34] J. P. Teifke and C. V. Löhr, "Immunohistochemical detection of P53 overexpression in paraffin wax-embedded squamous cell carcinomas of cattle, horses, cats and dogs," Journal of Comparative Pathology, vol. 114, no. 2, pp. 205-210, 1996.

[35] G. Sironi, P. Riccaboni, L. Mertel, G. Cammarata, and D. E. Brooks, "p53 protein expression in conjunctival squamous cell carcinomas of domestic animals," Veterinary Ophthalmology, vol. 2, no. 4, pp. 227-231, 1999.

[36] J. Reszec, M. Sulkowska, M. Koda, L. Kanczuga-Koda, and S. Sulkowski, "Expression of cell proliferation and apoptosis markers in papillomas and cancers of conjunctiva and eyelid," Annals of the New York Academy of Sciences, vol. 1030, pp. 419426, 2004.

[37] J. Gerdes, L. Li, C. Schlueter et al., "Immunobiochemical and molecular biologic characterization of the cell proliferationassociated nuclear antigen that is defined by monoclonal antibody Ki-67," American Journal of Pathology, vol. 138, no. 4, pp. 867-873, 1991.

[38] G. Singh-Ranger and K. Mokbel, "The role of cyclooxygenase2 (COX-2) in breast cancer, and implications of COX-2 inhibition," European Journal of Surgical Oncology, vol. 28, no. 7, pp. 729-737, 2002.

[39] C. S. Williams, M. Mann, and R. N. DuBois, "The role of cyclooxygenases in inflammation, cancer, and development," Oncogene, vol. 18, no. 55, pp. 7908-7916, 1999.

[40] S. I. Mohammed, D. Dhawan, S. Abraham et al., "Cyclooxygenase inhibitors in urinary bladder cancer: in vitro and in vivo effects," Molecular Cancer Therapeutics, vol. 5, no. 2, pp. 329336, 2006. 

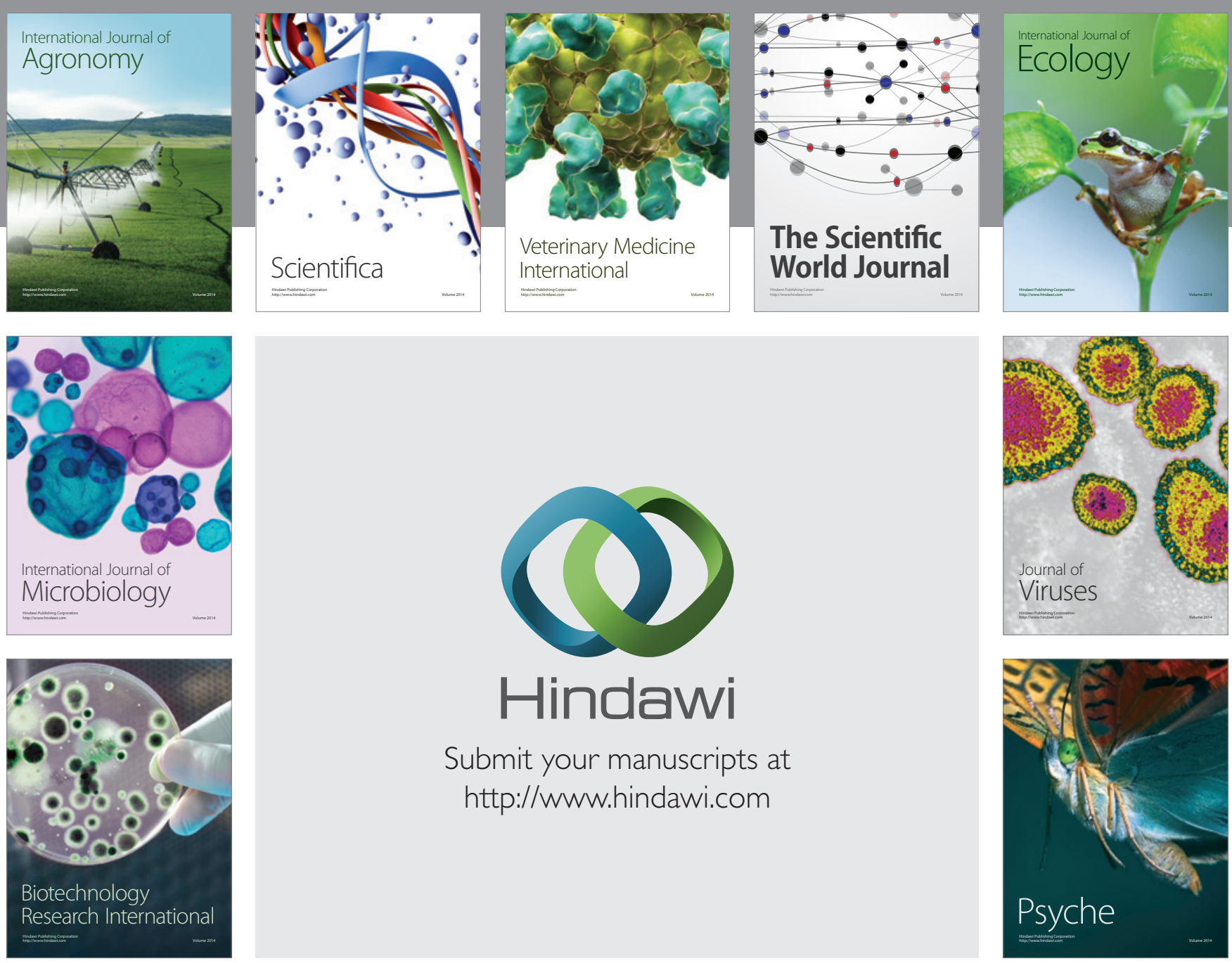

Submit your manuscripts at

http://www.hindawi.com
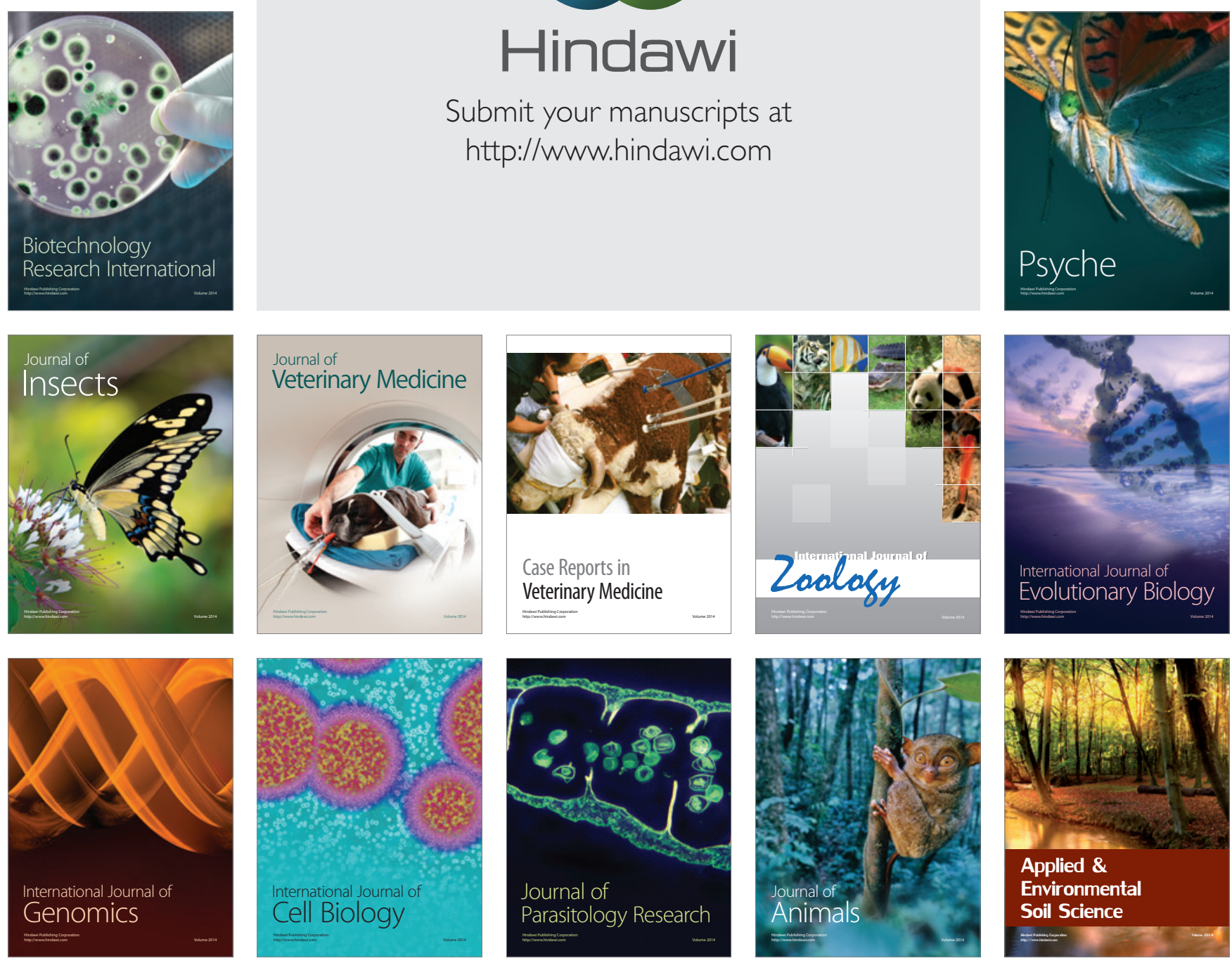